\title{
The Effects of Bilirubin and Phototherapy on Neonatal Thiol-Disulfide Homeostasis
}

\author{
Sara Erol1, Zehra Arslan33, Dilek Ulubas Isik33, Ahmet Yagmur Bas1, Nihal Demirel1 and Ozcan Erel2 \\ IDepartment of Neonatology / Clinical Biochemistry², Faculty of Medicine, Yildirim Beyazit University, Ankara, Turkey \\ 3Department of Neonatology, Etlik Zubeyde Hanim Women's Health Teaching and Research Hospital, Ankara, Turkey
}

\begin{abstract}
Objective: To determinate the effects of bilirubin and phototherapy on oxidative stress in newborns.

Study Design: A case-control study.

Place and Duration of Study: Third level Newborn Intensive Care Unit, Ankara Etlik Zubeyde Hanim Women's Health Teaching and Research Hospital, Turkey, from May to August 2017.

Methodology: Blood samples of 62 term newborns were grouped as control, before and after phototherapy. Total and native thiol, disulfide and ischemia modified albumin values in expressed blood samples were measured. Disulfide-native thiol ratio, disulfide-total thiol ratio and native thiol-total thiol ratio values were computed.

Results: Bilirubin levels were positively correlated with native and total thiol levels and negatively correlated with ischemia modified albumin levels $(r=0.409 p=0.001, r=0.328 p<0.009, r=-0.503 p<0.001)$. Native and total thiol levels of the control group were lower $(p<0.001)$ and ischemia modified albumin levels were higher than those before and after phototherapy $(p<0.001)$. In jaundiced newborns, native and total thiol values reduced after phototherapy, while IMA levels increased $(p=0.001, p<0.001, p<0.001)$.

Conclusion: Bilirubin showed antioxidant effect without increasing oxidative stress. Oxidative stress increased after phototherapy. This result was associated with decrease in bilirubin rather than oxidative effect of phototherapy.
\end{abstract}

Key Words: Newborn jaundice, Oxidative stress, Thiol-disülfide.

How to cite this article: Ero IS, Arslan Z, Isik DU, Bas AY, Demirel N, Erel O. The effects of bilirubin and phototherapy on neonatal thioldisulfide homeostasis. J Coll Physicians Surg Pak 2019; 29(9):843-7.

\section{INTRODUCTION}

Antioxidant defence systems regulate the increase of endogen and exogenous reactive oxygen products in the human body. ${ }^{1}$ These systems have not adequately protective effects on oxidative stress in neonates. As a result of insufficient antioxidant effects, lipid peroxidation and protein oxidation occur. ${ }^{2}$ Reactive oxygen products are responsible for the development of severe neonatal diseases such as retinopathy of prematurity, bronchopulmonary dysplasia, periventricular leukomalacia, intraventricular hemorrhage, sepsis, necrotizing enterocolitis, hypoxic ischemic encephalopathy. ${ }^{3}$ In recent years, the effects of newborn diseases on the oxidative system have been investigated by using various oxidative markers.

There are many studies demonstrating the effects of both hyperbilirubinemia and phototherapy used for the treatment of hyperbilirubinemia on the oxidative balance of neonates. ${ }^{4}$ Some studies conclude that bilirubin has

Correspondence to: Dr. Sara Erol, Department of Neonatology, Faculty of Medicine, Yildirim Beyazit University 06031,

Ankara, Turkey

E-mail: sarasurmeli@gmail.com

Received: February 06, 2019; Revised: May 16, 2019; Accepted: June 26, 2019 anti-oxidant effects in neonates. ${ }^{5}$ However, there are also clinical studies showing that bilirubin has prooxidant effects or no effect on oxidative balance of newborns. 6,7 In addition, some clinical trials show that oxidative stress markers increase after phototherapy. ${ }^{8}$ Consequently, understanding the effects of hyperbilirubinemia on oxidative balance becomes more complicated.

The thiol-disulfide homeostasis is a new oxidative marker. ${ }^{9}$ Thiol is one of the most important antioxidant mechanisms. A significant portion of the thiols in plasma is composed of protein thiols such as albumin. A smaller proportion is lower weight thiols such as glutathione, cysteine, homocysteine and gamma glutamine.10 Thiol neutralises reactive oxygen products. Sulfhydryl groups in the structure of thiol are oxidised to disulfide bonds. Disulfide bonds are converted to thiol again. In case of oxidative stress, the balance shifts to disulfide direction. ${ }^{11}$ Dynamic thiol-disulfide homeostasis may provide information about changing biochemical processes in diseases. ${ }^{9}$

The primary aim of this study was to determine the effects of hyperbilirubinemia and phototherapy on oxidative status. The secondary aim of this study was to investigate the correlation of thiol-disulfide homeostasis with serum ischemia modified albumin (IMA) levels, which were accepted as an oxidative marker. 


\section{METHODOLOGY}

This case-control study was performed in the third-level Neonatal Intensive Care Unit (NICU), Ankara Etlik Zubeyde Hanim Women's Health Teaching and Research Hospital, Turkey. Local ethics committee permission $(2016 / 3)$ and informed consent from families were obtained before the study started. The study was completed between 1 May 2017 and 1 August 2017 in 3-month period.

Term neonates, who were admitted to NICU for phototherapy, were included in this study. Infants exposed to perinatal hypoxia, infants who were received inotropes or oxygen support and had major congenital anomalies, direct hyperbilirubinemia, intracranial hemorrhage or infection, received blood transfusion, were excluded thereafter. The control group consisted of healthy term newborns at postnatal first week, who needed blood collection for other reasons such as blood group, thyroid function tests, complete blood count during outpatient visits.

Blood samples were obtained for the complete blood count, total serum bilirubin and albumin levels from infants with jaundice. Phototherapy was started in patients with high total serum bilirubin levels according to American Academy of Pediatrics guidelines. Phototherapy was not interrupted except when the mother was breastfeeding. Bilirubin was measured again 12 hours after phototherapy discontinuation.

Gestational age, birth weight, gender, mode of delivery, blood type of the baby and mother, postnatal time of phototherapy, and duration of phototherapy were recorded. Thiol-disulfide homeostasis was studied by an automated method that was developed by Erel and colleagues. ${ }^{9}$ Firstly, functional thiol groups are formed by the reduction of the dynamic disulfide bonds, in the plasma with sodium borohydride $(\mathrm{NaBH} 4)$. Unused $\mathrm{NaBH} 4$ residues are removed by formaldehyde to prevent the depletion of disulfide bonds formed after reaction with 5, 5'- dithiobis-2-nitrobenzoic acid (DTNB). The total thiol level in the sample was determined using modified Ellman reagent. When the natural thiol content is removed from the total thiol content, half of the result obtained determines the amount of disulfide bond. The unit of measurement for native thiol, total thiol and disulfide was $\mu \mathrm{moll} / \mathrm{Lt}$. Disulfide-native thiol ratio, disulfide-total thiol ratio, and native thiol-total thiol ratio values were computed and expressed as percentage.

The method developed by Bar-Or et al. was used for the determination of ischemia modified albumin levels. ${ }^{12}$ This is a fast colorimetric method. The difference of absorbance units between control and dithiothreitol samples was recorded. The results were quantified as absorbance unit (ABS Unit).

In the case group, serum thiol-disulfide homeostasis and serum IMA levels were evaluated before the onset of phototherapy and 12 hours after the end of photo- therapy. In the control group, thyol-disulfide homeostasis and ischemia modified albumin levels were measured from a single sample. Two $\mathrm{ml}$ of extra blood was collected for the samples and centrifuged at $3600 \mathrm{rpm}$ during 10 mins. The obtained plasma samples were stored at $-80^{\circ} \mathrm{C}$ in the eppendorf tube.

SPSS 15 package programme (SPSS Inc., Chicago, IL) was used for statistical analysis. Shapiro-Wilk test was used to determine the distribution forms of the variables. Numeric values were indicated as median (interquartile range) or mean \pm standard deviation, where appropriate and categorical values were indicated as $n(\%)$. Chisquare test was applied for categorical variables. The relationship between parametric variables was evaluated by Pearson analysis; and nonparametric variables were evaluated by Spearman correlation. Paired sample t-test for parametric variables was used to compare continuous variables in two dependent groups. Independent samples t-test for parametric variables and MannWhitney U-test for nonparametric variables were applied to compare variables in two independent groups. Results with $p<0.05$ were considered statistically significant.

\section{RESULTS}

Sixty-two term neonates were included in this study. The case group consisted of 31 infants who received phototherapy in our Neonatal Intensive Care Unit and met the study criteria. The control group consisted of 31 healthy infants whose blood samples were taken in

Table I: Comparison of clinical characteristics of case and control groups.

\begin{tabular}{|c|c|c|c|}
\hline & $\begin{array}{l}\text { Control group } \\
\qquad(n=31)\end{array}$ & $\begin{array}{c}\text { Phototherapy group } \\
(n=31)\end{array}$ & $\mathrm{p}$-values \\
\hline \multicolumn{4}{|l|}{ Gender $^{1}$} \\
\hline Female, n (\%) & $17(55)$ & $14(45)$ & 0.446 \\
\hline Male, n (\%) & $14(45)$ & $17(55)$ & \\
\hline \multicolumn{4}{|l|}{ Type of delivery 1} \\
\hline Vaginal delivery, n (\%) & $15(48)$ & $16(52)$ & 0.799 \\
\hline Cesarean section, $\mathrm{n}(\%)$ & $16(52)$ & $15(48)$ & \\
\hline Birth weight ${ }^{2}$, gram & $3322 \pm 320.93$ & $3141 \pm 493.52$ & 0.092 \\
\hline Gestational week ${ }^{3}$, weeks & $39(37-40)$ & $38(37-42)$ & 0.114 \\
\hline
\end{tabular}

1: Chi-square test: Categorical values are expressed as number and \%.

2: Independent samples t-test: values are expressed as mean $\pm S D$.

3: Mann-Whitney $U$ test: values are expressed as median (minimum-maximum).

Table II: Comparison of biochemical parameters before and after phototherapy.

\begin{tabular}{l|c|c|c}
\hline & $\begin{array}{c}\text { Before } \\
\text { phototherapy } \\
\mathrm{N}=31\end{array}$ & $\begin{array}{c}\text { After } \\
\text { phototherapy } \\
\mathrm{N}=31\end{array}$ & p-values \\
\hline Albumin, gr/Lt & $2.98 \pm 0.32$. & $2.76 \pm 0.23$ & 0.001 \\
Bilirubin, $\mathrm{mg} / \mathrm{dl}$ & $18.18 \pm 3.24$ & $11.14 \pm 2.31$ & 0.000 \\
Native-thiol, $\mu \mathrm{mol} / \mathrm{Lt}$ & $294.40 \pm 52.75$ & $243.42 \pm 48.83$ & 0.001 \\
Total-thiol, $\mu \mathrm{mol} / \mathrm{Lt}$ & $341.88 \pm 53.94$ & $288.26 \pm 47.28$ & 0.000 \\
Disulfide, $\mu \mathrm{mol} / \mathrm{Lt}$ & $23.74 \pm 9.57$ & $22.41 \pm 10.07$ & 0.597 \\
Disulfide:native-thiol ratio, (\%) & $8.39 \pm 3.93$ & $9.95 \pm 6.94$ & 0.302 \\
Disulfide:total-thiol ratio, (\%) & $7 \pm 2.81$ & $7.87 \pm 3.93$ & 0.345 \\
Native-thiol:total-thiol ratio, (\%) & $85.99 \pm 5.63$ & $84.25 \pm 7.87$ & 0.345 \\
Ischemia modified albumin & $0.63 \pm 0.06$ & $0.70 \pm 0.053$ & 0.000 \\
(ABS Unit) & & & \\
\hline
\end{tabular}


Table III: Biochemical comparison of control group and patient group.

\begin{tabular}{|c|c|c|c|c|c|c|}
\hline & \multicolumn{3}{|c|}{ Control group vs Before phototherapy group } & \multicolumn{3}{|c|}{ Control group vs After phototerapy group } \\
\hline & $\begin{array}{l}\text { Control group } \\
\qquad N=31\end{array}$ & $\begin{array}{l}\text { Before phototerapy } \\
\qquad N=31\end{array}$ & $p$-values & $\begin{array}{l}\text { Control group } \\
\qquad N=31\end{array}$ & $\begin{array}{c}\text { After phototherapy } \\
\qquad \mathrm{N}=31\end{array}$ & p-values \\
\hline Albumin, gr/Lt & $3.70 \pm 0.42$ & $2.99 \pm 0.32$ & 0.000 & $3.70 \pm 0.42$ & $2.76 \pm 0.23$ & 0.000 \\
\hline Native-thiol, $\mu \mathrm{mol} / \mathrm{Lt}$ & $204.97 \pm 32.97$ & $294.40 \pm 52.75$ & 0.000 & $204.97 \pm 32.97$ & $243.42 \pm 48.83$ & 0.001 \\
\hline Total-thiol, $\mu \mathrm{mol} / \mathrm{Lt}$ & $245.31 \pm 40.92$ & $341.88 \pm 53.94$ & 0.000 & $245.31 \pm 40.92$ & $288.26 \pm 47.28$ & 0.000 \\
\hline Disulfide, $\mu \mathrm{mol} / \mathrm{Lt}$ & $20.17 \pm 7.35$ & $23.74 \pm 9.57$ & 0.105 & $20.17 \pm 7.35$ & $22.41 \pm 10.07$ & 0.320 \\
\hline Disulfide: native-thiol ratio (\%) & $9.89 \pm 3.37$ & $8.39 \pm 3.93$ & 0.113 & $9.89 \pm 3.37$ & $9.95 \pm 6.94$ & 0.964 \\
\hline Disulfide: total-thiol ratio (\%) & $8.12 \pm 2.37$ & $7 \pm 2.81$ & 0.094 & $8.12 \pm 2.37$ & $7.87 \pm 3.93$ & 0.756 \\
\hline Native-thiol: total-thiol ratio (\%) & $83.74 \pm 4.75$ & $85.99 \pm 5.63$ & 0.094 & $83.74 \pm 4.75$ & $84.25 \pm 7.87$ & 0.756 \\
\hline Ischemia modified albumin (ABSUnit) & $0.84 \pm 0.09$ & $0.63 \pm 0.06$ & 0.000 & $0.84 \pm 0.09$ & $0.70 \pm 0.053$ & 0.000 \\
\hline
\end{tabular}

outpatient visits. The case group and the control group were similar in terms of gender, gestational week, birth weight, and type of delivery (Table I).

When the biochemical parameters before and after phototherapy were compared, serum albumin and bilirubin levels were significantly decreased after phototherapy $(p=0.001 p<0.001)$. Moreover, native thiol and total thiol levels were significantly lower after phototherapy $(p=0.001 p<0.001)$. IMA values had significantly increased after phototherapy $(p<0.001$, Table II). The mean duration of phototherapy was 34.9 (24-72) hours. There was no correlation between the exposure time to phototherapy and serum IMA, native thiol, total thiol and disulfide values.

The biochemical parameters of the control group were compared with the values before and after phototherapy. In the control group, the levels of native thiol and total thiol were found to be lower than the values before and after the phototherapy (for native thiol $p<0.001 p=0.001$, for total thiol $p<0.001, p<0.001)$. There was no significant difference between the cases and the controls in terms of disulfide values and disulfide-native thiol ratio, disulfide-total thiol ratio, and native thiol-total thiol ratio. Albumin and IMA levels of control group were significantly higher than case group $(p<0.001, p<0.001$, Table III).

The correlation of biochemical data with each other was examined. Bilirubin levels were positively correlated with albumin, total thiol and native thiol levels $(r=0.360 p=$ $0.004, r=0.409 p=0.001, r=0.328 p=0.009$ ). However, negative correlations were found between bilirubin and IMA levels $(r=-0.503, p<0.001)$. Serum IMA levels were negatively correlated with native and total thiol levels $(r=-0.258 p=0.043, r=-0.325 p=0.010)$. Disulfide levels were not correlated with bilirubin, albumin, IMA, total and native thiol levels.

\section{DISCUSSION}

Hyperbilirubinemia is a clinical problem that may have potential toxic effects on the central nervous system in both term and preterm neonates. ${ }^{13}$ The anti-oxidant effect of bilirubin is clearly demonstrated in laboratory experiment and animal studies.14,15 There are many clinical studies showing that toxic oxygen products and free radicals are reduced by bilirubin. ${ }^{16}$ The effects of bilirubin on term and preterm infants are still controversial.

The relationships between bilirubin and serum malondialdehyde, ascorbate, urate and alpha - tocopherol levels, trolox equivalents, superoxide dismutas, glutathione peroxidase and catalase activities have evaluated in different clinical studies in term infants. ${ }^{4}$ In some of these studies, high levels of bilirubin have shown as a reason of oxidative stress. ${ }^{6}$ The prooxidant effects of bilirubin have attributed to different mechanisms such as overstimulation of glutamate receptors, increased proinflammatory cytokines and activity of neuronal nitric oxide synthase.17,18 However, some clinical trials have demonstrated the antioxidant effect of bilirubin. ${ }^{19}$ These clinical studies have showed that the high bilirubin levels did not increase oxidative stress and lipid peroxidation unless additional risk conditions such as low albumin level, glucose-6-phosphate dehydrogenase deficiency, and hepatic glucuronyl transferase deficiency were not presented. 19 In this study, bilirubin levels were positively correlated with albumin, total thiol and native thiol levels, and negatively correlated with IMA. In the case of oxidative conditions, the amount of thiol is reduced and the thiol - disulfide balance shifts to disulfide direction. According to these results, the positive correlation between bilirubin and thiol levels was attributed to antioxidant effect of bilirubin. Similarly, the absence of a relationship between bilirubin and disulfide levels may suggest that bilirubin has no effect on oxidative damage. Negative correlation of bilirubin with IMA is supported this opinion. In the present study, native and total thiol levels of the infants without jaundice in control group were lower than levels before and after phototherapy in case group. This result strengthened our arguments that bilirubin is an antioxidant for newborns. In addition, lower IMA levels in case group may be interpreted as protective effect of bilirubin from oxidative stress.

Phototherapy is considered safe in neonates despite side effects. However, recent studies have demonstrated the relationships between phototherapy and lipid peroxidation, oxidative stress and DNA damage. ${ }^{6}$ Some clinical studies have shown that conventional phototherapy increased oxidative stress more than LED 
phototherapy. 20 In another study, oxidative stress index after phototherapy has found to be increased independently of the light source. 21 The researches have shown that the antioxidant capacity of parenteral nutrition solutions and the mother's milk was decreased after exposure to phototherapy light.22,23 The effect of phototherapy on oxidant status is not clear in neonates with jaundice. Different studies in term newborns have revealed that superoxide dismutase enzyme increased after phototherapy in response to oxidative stress. ${ }^{24}$ There are also clinical trials that have shown reduction in non-enzymatic antioxidants such as uric acid and vitamin $\mathrm{C}$ after phototherapy. ${ }^{25}$ In this study, the authors evaluated reduction of thiol levels after phototherapy as a decrease in antioxidant effect and increase of IMA values after phototherapy as a presence of oxidative stress. No increase in disulfide levels despite reduced thiol levels may suggest that oxidative stress is caused by other mechanisms. IMA levels in this control patients were evaluated to be higher than before and after phototherapy. This can be attributed to the reduction of oxidative stress by bilirubin in neonatal jaundice. The lack of correlation of IMA, thiol and disulfide values with exposure time to phototherapy can support that phototherapy is not solely responsible for the oxidative effect.

The main limitation of this study is that the forces of phototherapy devices were not evaluated. The other one is that the oxidative status was assessed by thioldisulfide homeostasis, while the additional parameters were not used to determine the oxidative stress marker, such as malondialdehyde. In spite of these, evaluation of the new oxidative marker, thiol-disulfide homeostasis, and the correlation of this balance with another oxidative marker, IMA, are the positive aspects of this study.

\section{CONCLUSION}

In neonates with insufficient defence against oxidant agents, it is very important to evaluate the effects of a common clinical condition such as hyperbilirubinemia and a common treatment method such as phototherapy on oxidative balance. This study supports the antioxidant effect of bilirubin on newborns. The increase in oxidative stress after phototherapy may be combined effect of bilirubin decline and phototherapy.

\section{ETHICAL APPROVAL:}

This case-control study was performed in the third-level Neonatal Intensive Care Unit (NICU), Ankara Etlik Zubeyde Hanim Women's Health Teaching and Research Hospital, Turkey. Local ethics committee permission $(2016 / 3)$

\section{PATIENTS' CONSENT:}

informed consent from families were obtained before the study started.

\section{CONFLICT OF INTEREST:}

Authors declared no conflict of interest.

\section{AUTHORS' CONTRIBUTION:}

SE: Designed, directed and coordinated the study, created study plan, analysed the data and written the article.

ZA, DUI: Selected patients and collected samples.

AYB, ND: Analysed the data.

OE: Studied the collected samples in biochemistry laboratory.

\section{REFERENCES}

1. Buonocore G1, Perrone S, Tataranno ML. Oxygen toxicity: Chemistry and biology of reactive oxygen species. Semin Fetal Neonatal Med 2010; 15:186-90.

2. Negi R, Pande D, Kumar A, Khanna RS, Khanna HD. Evaluation of biomarkers of oxidative stress and antioxidant capacity in the cord blood of preterm low birth weight neonates. Matern Fetal Neonatal Med 2012; 25:1338-41.

3. Saugstad OD. Update on oxygen radical disease in neonatology. Curr Opin Obstet Gynecol 2001; 13:147-53.

4. Dani C, Poggi C, Pratesi S. Bilirubin and oxidative stress in term and preterm infants. Free Radic Res 2018; 16:1-151.

5. Kumar A, Pant P, Basu S, Rao GR, Khanna HD. Oxidative stress in neonatal hyperbilirubinemia. $J$ Trop Pediatr 2007; 53: 69-71.

6. Basu S, De D, Dev Khanna H, Kumar A. Lipid peroxidation, DNA damage and total antioxidant status in neonatal hyperbilirubinemia. J Perinatol 2014; 34:519-23.

7. Gopinathan V, Miller NJ, Milner AD, Rice-Evans CA. Bilirubin and ascorbate antioxidant activity in neonatal plasma. FEBS Lett 1994; 349:197-200.

8. Ayyappan S, Philip S, Bharathy N, Ramesh V, Kumar CN, Swathi S, et al. Antioxidant status in neonatal jaundice before and after phototherapy. J Pharm Bioallied Sci 2015; 7:S16-21.

9. Erel O, Neselioglu S. A novel and automated assay for thiol/ disulphide homeostasis. Clin Biochem 2014; 47:326-32.

10. Turell L, Radi R, Alvarez B. The thiol pool in human plasma: The central contribution of albumin to redox processes. Free Radic Biol Med 2013; 65:244-53.

11. Cremers CM, Jakob U. Oxidant sensing by reversible disulfide bond formation. J Biol Chem 2013; 288:26489-96.

12. Bar-Or D, Lau E, Winkler JV. A novel assay for cobalt-albumin binding and its potential as a marker for myocardial ischemia: A preliminary report. J Emerg Med 2000; 19:311-5.

13. Shapiro SM. Bilirubin toxicity in the developing nervous system. Pediatr Neurol 2003; 29:410-21.

14. Kumagai $A$, Ando $R$, Miyatake $H$, Greimel $P$, Kobayashi $T$, Hirabayashi $Y$, et al. A bilirubin-inducible fluorescent protein from eel muscle. Cell 2013; 153:1602-11.

15. Worou ME, Belmokhtar K, Bonnet P, Vourc'h P, Machet MC, Khamis $\mathrm{G}$, et al. Hemin decreases cardiac oxidative stress and fibrosis in a rat model of systemic hypertension via PI3K/Akt signalling. Cardiovasc Res 2011; 91:320-9.

16. Gazzin S, Vitek L, Watchko J, Shapiro SM, Tiribelli C. A novel perspective on the biology of bilirubin in health and disease. Trends Mol Med 2016; 22:758-68. 
17. Brito MA, Vaz AR, Silva SL, Falcão AS, Fernandes A, Silva RF, et al. N-methyl-aspartate receptor and neuronal nitric oxide synthase activation mediate bilirubin-induced neurotoxicity. Mol Med 2010; 16:372-80.

18. Fernandes A, Falcao AS, Silva RF, Brito MA, Brites D. MAPKs are key players in mediating cytokine release and cell death induced by unconjugated bilirubin in cultured rat cortical astrocytes. Eur J Neurosci 2007; 25:1058-68.

19. Shekeeb Shahab M, Kumar P, Sharma N, Narang A, Prasad R. Evaluation of oxidant and antioxidant status in term neonates: a plausible protective role of bilirubin. Mol Cell Biochem 2008; 317:51-9.

20. Demirel G, Uras N, Celik IH, Aksoy HT, Oguz SS, Erdeve O, et al. Comparison of total oxidant/antioxidant status in unconjugated hyperbilirubinemia of newborn before and after conventional and LED phototherapy: A prospective randomized controlled trial. Clin Invest Med 2010; 33:E335-41.
21. Kale Y, Aydemir O, Celik Ü, Kavurt S, Isikoglu S, Bas AY, et al. Effects of phototherapy using different light sources on oxidant and antioxidant status of neonates with jaundice. Early Hum Dev 2013; 89:957-60.

22. Smith JL, Canham JE, Wells PA. Effect of phototherapy light, sodium bisulfite, and $\mathrm{pH}$ on vitamin stability in total parenteral nutrition admixtures. J Parenter Enteral Nutr 1988; 12:394-402.

23. Unal S, Demirel N, Yaprak Sul D, Ulubas Isik D, Erol S, Neselioglu $\mathrm{S}$, et al. The consequence of phototherapy exposure on oxidative stress status of expressed human milk. J Matern Fetal Neonatal Med 2019; 32:46-50.

24. Dahiya K, Tiwari AD, Shankar V, Kharb S, Dhankhar R. Antioxidant status in neonatal jaundice before and after phototherapy. Indian J Clin Biochem 2006; 21:157-60.

25. Aycicek A, Erel O. Total oxidant/antioxidant status in jaundiced newborns before and after phototherapy. J Pediatr (Rio J) 2007; 83:319-22. 